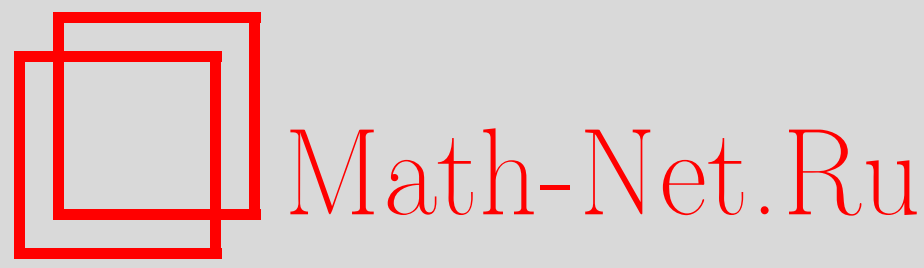

А. А. Липчюс, Одно свойство независимых отображений, Теория вероятн. и ее примен., 2004, том 49, выпуск 2, 362-365

DOI: https://doi.org/10.4213/tvp225

Использование Общероссийского математического портала Math-Net.Ru подразумевает, что вы прочитали и согласны с пользовательским соглашением

http: //www . mathnet.ru/rus/agreement

Параметры загрузки:

IP : 54.196 .121 .252

26 апреля 2023 г., $15: 39: 31$

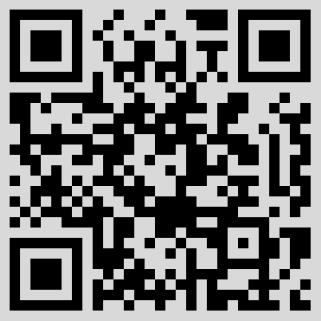


21. Seiffert I., Riedel M. Uniqueness of two factorization problems. - Теория вероятн. и ее примен., 1979 , т. 24 , в. 1, с. 207-211.

22. Smirnoff $V$. Sur les valeurs limites des fonction régulières à l'intérieur d'un cercle. Журн. Ленинград. физ.-матем. об-ва, 1928, т. 2, № z, с. 22-37.

23. Walther $A$. The question of phase retrieval in optics. - Optica Acta, 1963, v. 10, p. $41-49$.

Поступила в редакцию

28.I.2004

(c) $2004 \mathrm{r}$.

ЛИПЧЮС А. А.*

\section{ОДНО СВОЙСТВО НЕЗАВИСИМЫХ ОТОБРАЖЕНИЙ ${ }^{1)}$}

В статье рассматривается достаточное условие (справедливое в случае общих вероятностных пространств) того, что с данным отображением могут быть независимы только конечнозначные случайные величины.

Ключевые слова и фразы: независимость, конечное число значений, теорема Оттавиани, абсолютная непрерывность.

Целью данной работы является обобщение следуюшего результата, полученного в 1947 году Дж. Оттавиани [1]. Пусть $f, g:[0,1] \rightarrow \mathbf{R}$ - измеримые функции, $g$ абсолютно непрерывна и отлична от константы, причем $f$ и $g$ независимы как случайные величины на отрезке с мерой Лебега. Тогда $f$ почти всюду совпадает с функцией, принимающей конечное множество значений.

Естественно возникает вопрос, какие свойства отображения $g$ отвечают за то, что с ним могут быть независимы только ступенчатые функции с конечным числом значений. Оказывается, что для общих вероятностных пространств имеет место следующий более общий результат, в котором независимость отображений $f$ и $g$ со значениями в измеримом пространстве $(X, \mathscr{A})$ понимается в обычном смысле: $\mathbf{P}\{f \in A, g \in B\}=\mathbf{P}\{f \in A\} \mathbf{P}\{g \in B\}$ для всех $A, B \in \mathscr{A}$. Через $\mathbf{P} \circ g^{-1}$ будем обозначать образ меры $\mathbf{P}$ при отображении $g$, т.е. $\mathbf{P} \circ g^{-1}(B)=\mathbf{P}\left(g^{-1}(B)\right)$. Напомним, что $\sigma$-алгебра $\mathscr{A}$ называется счетно-порожденной, если она порождается не более чем счетным семейством множеств. Если существует не более чем счетное семейство множеств $A_{n} \in \mathscr{A}$, разделяющих точки $X$ (т.е. для всякой пары различных точек $x, y \in X$ найдется такое $A_{n}$, что либо $x \in A_{n}, y \notin A_{n}$, либо $y \in A_{n}, x \notin A_{n}$ ), то $\mathscr{A}$ называется счетно-разделяющей. Множество $E$ называется измеримым относительно меры $\nu$ на $\mathscr{A}$, если оно входит в лебеговское пополнение $\mathscr{A}$ относительно $\nu$.

Теорема 1. Пусть $(\Omega, \mathscr{F}, \mathbf{P})$ - вероятностное пространство, $(X, \mathscr{A})$ - измеримое пространство со счетно-порожденной и счетно-разделяющей $\sigma$-алгеброй $\mathscr{A}$. Предположим, что отображения $f, g: \Omega \rightarrow X$ измеримы и независимы, причем $g$ удовлетворяет следующим двум условиям:

1) $g(E)$ измеримо относительно меры $\mathbf{P} \circ g^{-1}$ для всех $E \in \mathscr{F}$;

2) для всякой последовательности попарно непересекаюиихся множеств $A_{k} \in \mathscr{F}$ c $\mathbf{P}\left(A_{k}\right)>0$ u $\lim _{k \rightarrow \infty} \mathbf{P}\left(A_{k}\right)=0$ cyществует maкое $n$, ито $\mathbf{P} \circ g^{-1}\left(g\left(A_{n}\right)\right)<1$.

Тогда $f$ почти всюду совпадает с отображением, принимающим конечное множество значений.

* Московский государственный университет им. М. В. Ломоносова, механико-математический факультет, Ленинские горы, 119899 Москва, Россия.

1) Работа выполнена при частичной поддержке проекта РФФИ 04-01-00748. 
Д о к а з а т л ь с т в о. Так как любое измеримое пространство со счетнопорожденной и счетно-разделяющей $\sigma$-алгеброй изоморфно некоторому подмножеству в $[0,1]$ с борелевской $\sigma$-алгеброй $\mathscr{B}(M)$ (см. [2, теорема 6.5.8]), то можно считать, что $f, g: \Omega \rightarrow M \subset[0,1], \mathscr{A}=\mathscr{B}(M)$. Докажем следующее вспомогательное утверждение: если $A \in \mathscr{F}, \mathbf{P}(A)>0$ и $g$ независима с $I_{A}$, то $\mathbf{P} \circ g^{-1}(g(A))=1$. Действительно,

$$
0=\mathbf{P}\left\{g \in X \backslash g(A), I_{A}=1\right\}=\mathbf{P}\{g \in X \backslash g(A)\} \mathbf{P}(A),
$$

откуда $\mathbf{P}\{g \in X \backslash g(A)\}=0$, т.е. $\mathbf{P}\{g \in g(A)\}=1$. Отметим, что в этом вычислении мы воспользовались определяющим соотношением независимости $g$ и $I_{A}$ применительно к множеству $E=X \backslash g(A)$, которое может и не входить в $\mathscr{B}(M)$. Чтобы обосновать это, заметим, что по условию найдутся такие множества $B_{1}, B_{2} \in \mathscr{B}(M)$, что $B_{1} \subset E \subset B_{2}$ и $\mathbf{P} \circ g^{-1}\left(B_{1}\right)=\mathbf{P} \circ g^{-1}\left(B_{2}\right)$. Поэтому $g^{-1}\left(B_{1}\right) \subset g^{-1}(E) \subset g^{-1}\left(B_{2}\right)$, откуда вытекает, что $g^{-1}(E)$ измеримо относительно $\mathbf{P}$ и имеет равную с $g^{-1}\left(B_{1}\right)$ и $g^{-1}\left(B_{2}\right)$ P-меру. При этом

$$
\mathbf{P}\left\{g \in X \backslash g(A), I_{A}=1\right\}=\mathbf{P}\left\{g \in B_{1}, I_{A}=1\right\}=\mathbf{P}\left\{g \in B_{1}\right\} \mathbf{P}(A)=\mathbf{P}\{g \in E\} \mathbf{P}(A) .
$$

Напомним, что число $x$ называется существенным значением измеримого отображения $f$ в $\mathbf{R}$, если для всякого $\varepsilon>0$ имеем $\mathbf{P}\{f \in(x-\varepsilon, x+\varepsilon)\}>0$. Теперь докажем, что множество существенных значений отображения $f$ конечно. Предположим противное, т.е. что имеется счетное число существенных значений $\left\{x_{i}\right\}_{i=1}^{\infty}$. Будем строить последовательность множеств $A_{n} \in \mathscr{F}$ таких, что они имеют положительные стремящиеся к нулю меры, $g$ независима с каждым $I_{A_{n}}$. Положим $U_{\varepsilon}(x):=(x-\varepsilon, x+\varepsilon)$. По определению существенного значения существует такое $\varepsilon_{1,1}>0$, что $\mathbf{P}\left\{f \in U_{\varepsilon_{1,1}}\left(x_{1}\right)\right\}>0$. Положим $A_{1}:=f^{-1}\left(U_{\varepsilon_{1,1}}\left(x_{1}\right)\right)$. Сушествуют такие $\varepsilon_{1,2}, \varepsilon_{2,2}>0$, что $U_{\varepsilon_{1,2}}\left(x_{1}\right) \cap U_{\varepsilon_{2,2}}\left(x_{2}\right)=\varnothing$. Существует $k \in\{1,2\}$ такое, что $0<\mathbf{P}\left\{f \in U_{k, 2}\right\}<\frac{1}{2}$. Положим $A_{2}=f^{-1}\left(U_{\varepsilon_{k, 2}}\left(x_{k}\right)\right)$. Продолжая индуктивно, на $n$-м шаге получаем, что существуют такие $\varepsilon_{1, n}, \ldots, \varepsilon_{n, n}>0$, что $U_{\varepsilon_{i, n}}\left(x_{i}\right)$ попарно не пересекаются, $i=1, \ldots, n$, а также существует такое $k \in\{1, \ldots, n\}$, что $0<\mathbf{P}\left\{f \in U_{\varepsilon_{k, n}}\left(x_{k}\right)\right\}<n^{-1}$. Положим $A_{n}=f^{-1}\left(U_{\varepsilon_{k, n}}\left(x_{k}\right)\right)$.

В силу построения и независимости $f$ и $g$ получили требуемую последовательность множеств $A_{n}$. Из построения видно, что $A_{k}$ можно было брать непересекаюшимися. Используя доказанное выше утверждение, имеем $\mathbf{P} \circ g^{-1}\left(g\left(A_{n}\right)\right)=1$ для всех $n$ - противоречие с условием.

Итак, доказано, что множество существенных значений $f$ конечно. Пусть $x_{1}, \ldots, x_{k}$ - все эти значения, т.е. других сушественных значений нет. Докажем, что

$$
\mathbf{P} \circ f^{-1}\left(M \backslash\left\{x_{1}, \ldots, x_{k}\right\}\right)=0 .
$$

Это и будет означать утверждение теоремы. Пусть $G=M \backslash\left\{x_{1}, \ldots, x_{k}\right\}$. Обозначим через $\tau$ топологию в $M$, индуцированную из $[0,1]$. Пусть $\left\{B_{i}\right\}_{i=1}^{\infty}-$ счетная база топологии $\tau$. По определению $G$ для всякого $x \in G$ существует такой интервал $U_{x} \in \tau$, что $x \in U_{x}$ и $\mathbf{P} \circ f^{-1}\left(U_{x}\right)=0$. Тогда $G=\bigcup_{k=1}^{\infty} B_{n_{k}}$, причем для каждого $k \in \mathbf{N}$ найдется такая точка $x \in G$, что $B_{n_{k}} \subset U_{x}$, откуда $\mathbf{P} \circ f^{-1}\left(B_{n_{k}}\right) \leqslant \mathbf{P} \circ f^{-1}\left(U_{x}\right)=0$. Таким образом, получаем $\mathbf{P} \circ f^{-1}(G)=\mathbf{P} \circ f^{-1}\left(\bigcup_{k=1}^{\infty} B_{n_{k}}\right) \leqslant \sum_{k=1}^{\infty} \mathbf{P} \circ f^{-1}\left(B_{n_{k}}\right)=0$,

Отметим, что условие 1) автоматически выполнено для борелевских отображений суслинских пространств, наделенных борелевскими $\sigma$-алгебрами. Поэтому в большинстве реально возникающих ситуаций остается лишь условие 2).

Теперь покажем, что теорема Оттавиани непосредственно вытекает из нашего результата. Известно (см. [2]), что борелевское отображение отрезка в $\mathbf{R}$ обладает тем свойством, что образы борелевских множеств универсально измеримы. Кроме того, абсолютно непрерывная функция на отрезке с мерой Лебега $\lambda$ обладает свойством (S) Банаха: для всякого $\varepsilon>0$ найдется $\delta>0$ такое, что как только $A \in \mathscr{B}([0,1])$ и $\lambda(A)<\delta$, то $\lambda(g(A))<\varepsilon$. Так как функция $g$ отлична от константы, то у меры $\mu \circ g^{-1}$ имеется компонента, абсолютно непрерывная относительно меры Лебега (таковой будет образ сужения меры Лебега на множество, где производная $g$ существует и отлична от нуля, см. [2, лемма 5.8.13]), а тогда в силу абсолютной непрерывности интеграла Лебега получаем, что $g$ удовлетворяет условиям теоремы Оттавиани. 
Получаем, что в теореме Оттавиани условие абсолютной непрерывности, накладываемое на функцию $g$, можно ослабить до следующих трех условий: измеримость образов борелевских множеств относительно меры, индуцированной $g$ (что автоматически выполнено для борелевских функций), свойство (S) Банаха и наличие у образа меры Лебега при отображении $g$ абсолютно непрерывной компоненты относительно меры Лебега. Заметим, что свойство (S) нельзя заменить на более слабое свойство (N) Лузина (образ всякого множества меры нуль имеет меру нуль). Это будет показано в следуюшем замечании.

3 а м е ч а н и е 1 . Если в теореме Оттавиани заменить отрезок $[0,1]$ на интервал $(0,1)$ и понимать абсолютную непрерывность как абсолютную непрерывность на каждом подотрезке, то теорема будет неверна. Пример: положим $g(x)=2^{2 k}\left(x-\alpha_{2 k}\right)$ при $x \in\left(\alpha_{2 k}, \beta_{2 k}\right]$ и $g(x)=2^{2 k-1}\left(\beta_{2 k-1}-x\right)$ при $x \in\left(\alpha_{2 k-1}, \beta_{2 k-1}\right]$, где

$$
\alpha_{1}=0, \quad \beta_{1}=\frac{1}{2} ; \quad \alpha_{k+1}=\beta_{k}, \quad \beta_{k+1}=\beta_{k}+\frac{1}{2^{k+1}}, \quad k=1,2, \ldots .
$$

Положим

$$
f=\sum_{k=1}^{\infty} k I_{\left(\alpha_{k}, \beta_{k}\right)}
$$

Тогда $g$ абсолютно непрерывна на каждом подотрезке, $f$ и $g$ независимы, у $f$ счетное множество существенных значений.

Этот же пример показывает, что свойство $(\mathrm{S})$ нельзя заменить на свойство $(\mathrm{N})$. Действительно, доопределим $f$ и $g$ на концах интервала, например, нулем. Тогда $g$ обладает свойством (N), так как она взаимно однозначна на каждом интервале $\left(\alpha_{k}, \beta_{k}\right)$, но не обладает свойством (S) в силу того, что образ интервала $(1-\varepsilon, 1)$ для всех $\varepsilon>0$ имеет меру 1 .

3 а м е ч а н и е 2. В теореме Оттавиани требование абсолютной непрерывности нельзя заменить на непрерывность и ограниченность вариации. Например, в качестве $g$ возьмем лестницу Кантора, которая является непрерывной функцией ограниченной вариации, но не абсолютно непрерывна. Дополнение к множеству Кантора счетное множество непересекающихся интервалов $\left\{\left(\alpha_{k}, \beta_{k}\right)\right\}_{k=1}^{\infty}$. Положим

$$
A_{n}=\bigcup_{k=1}^{\infty}\left(\alpha_{k}+\frac{\beta_{k}-\alpha_{k}}{2^{n}}, \alpha_{k}+\frac{\beta_{k}-\alpha_{k}}{2^{n-1}}\right) \quad \text { при } n=2,3, \ldots,
$$

$A_{1}=[0,1] \backslash \bigcup_{k=2}^{\infty} A_{k}$. Пусть $f=\sum_{k=1}^{\infty} k I_{A_{k}}$, тогда $f$ и $g$ независимы.

3 а м е ч а н и е 3 . Если в качестве $g$ в нашей теореме взять тождественное отображение $g:[0,1] \rightarrow[0,1], g(x)=x$, с борелевскими $\sigma$-алгебрами, то предположения теоремы будут выполнены, однако если изменить $g$ на множестве Кантора, а именно определить ее там как биекцию множества Кантора с отрезком $[0,1]$, которая сохраняет порядок, то первое условие сохранится, а второе нарушится. Этот пример показывает, что невыполнение второго условия еше не влечет существование функции, независимой с данной и принимающей хотя бы счетное множество существенных значений. Более того, так как свойство независимости двух отображений инвариантно относительно переопределения отображений на множестве меры нуль, то в нашей теореме достаточно потребовать от отображения $g$ наличия модификации с условиями 1) и 2). Однако остается открытым вопрос: если функция $g$ такова, что условие 2) не выполняется ни для какой ее версии, для которой выполнено условие 1), то всегда ли сушествует функция $f$, независимая с ней и принимаюшая счетное множество сушественных значений?

Этот же пример показывает, что если требовать выполнения второго условия нашей теоремы лишь для последовательностей непересекаюшихся множеств, то получится строго более слабое условие. Действительно, если в качестве $\left\{A_{k}\right\}_{k=1}^{\infty}$ взять покрытия множества Кантора интервалами со сколь угодно малой суммой длин, то условие 2) выполняться не будет, однако последовательности непересекаюшихся множеств с тем же свойством для данной функции нет. 


\section{СПИСОК ЛИТЕРАТУРЫ}

1. Ottaviani G. Sulla indipendenza delle funzioni misurabili. - Atti Accad. Naz. Lincei. Rend. Cl. Sci. Fis. Mat. Natur. (8), 1947, v. 2, p. 393-398.

2. Богачев В. И. Основы теории меры. Т. 1, 2. М.-Ижевск: Регулярная и хаотическая динамика, 2003.

Поступила в редакцию

21.IV.2004

(C) 2004 r.

HOBAK C. Ю.*

\section{О САМОНОРМИРОВАННЫХ СУММАХ СЛУЧАЙНЫХ ВЕЛИЧИН И СТАТИСТИКЕ СТЬЮДЕНТА}

В статье оценивается точность нормальной аппроксимации распределений некоторых нелинейных функционалов от сумм независимых случайных векторов. В качестве следствия получена оценка типа БерриЭссеена $c$ явными константами для распределений самонормированных сумм случайных величин и статистики Стьюдента.

Ключевые слова и фразы: самонормированные суммы случайных величин, статистика Стьюдента.

1. Введение. Задача оценивания точности нормальной аппроксимации распределений сумм независимых случайных величин восходит к работе А. М. Ляпунова [16]. Решение задачи в случае сумм с неслучайной нормировкой было найдено Э. Берри [5] и К.-Г. Эссееном [12]: если $X, X_{1}, X_{2}, \ldots$ - независимые одинаково распределенные случайные величины, $\mathbf{E} X=0$ и $\mathbf{E}|X|^{3}<\infty$, то

$$
\sup _{x}\left|\mathbf{P}\left\{\frac{\sum_{i=1}^{n} X_{i}}{\sigma \sqrt{n}}<x\right\}-\Phi(x)\right| \leqslant C_{*} n^{-1 / 2} \mathbf{E}|X|^{3} \sigma^{-3},
$$

где $C_{*}$ - абсолютная постоянная (известно [24], что $C_{*} \leqslant 0.7655$ ), $\sigma^{2}=\mathbf{D} X$ и $\Phi-$ функция распределения нормальной $\mathscr{N}(0 ; 1)$ случайной величины. В приложениях, как правило, дисперсия $\sigma^{2}$ не известна и должна быть заменена ее состоятельной оценкой $n^{-1} \sum_{i=1}^{n}\left(X_{i}-\widehat{X}_{n}\right)^{2}$, где $\widehat{X}_{n}=n^{-1} \sum_{i=1}^{n} X_{i}$ есть выборочное среднее. Таким образом, мы приходим к задаче оценивания точности нормальной аппроксимации для распределения статистики Стьюдента

$$
t_{n}=\sum_{i=1}^{n} X_{i}\left[\sum_{i=1}^{n}\left(X_{i}-\widehat{X}_{n}\right)^{2}\right]^{-1 / 2}
$$

Статистика Стьюдента известна своими многочисленными приложениями. Ее распределение изучалось в работах В. Бенткуса, Ф. Гётце, Э. Жине, Д. М. Мэйсона, Р. Фишера, П. Холла, К.-Л. Чжуна, Д. М. Чибисова, Б. Эфрона и других специалистов.

В. А. Егоров [28] и Э. Жине, Ф. Гётце и Д. М. Мэйсон [14] нашли необходимые и достаточные условия асимптотической нормальности $t_{n}$. В.В. Славова [23], применив результаты Чибисова [7], [8], показала, что

$$
\Delta_{n} \equiv \sup _{x}\left|\mathbf{P}\left\{t_{n}<x\right\}-\Phi(x)\right| \leqslant C n^{-1 / 2} \quad(\exists C<\infty) .
$$

* Middlesex University Business School, The Burroughs, London, NW4 4BT, UK; e-mail: s.novak@mdx.ac.uk 